\title{
Advancements in Imaging for Atrial Fibrillation Ablation: Is There a Potential to Improve Procedural Outcomes?
}

\author{
EDMOND OBENG-GYIMAH, MD ${ }^{1}$ and SAMAN NAZARIAN, MD, PhD ${ }^{1}$
}

\begin{abstract}
${ }^{1}$ Perelman Clinical Electrophysiology Section, Cardiovascular Division, Department of Medicine, School of Medicine at
\end{abstract} the University of Pennsylvania, Philadelphia, PA, USA

\begin{abstract}
Since the introduction of atrial fibrillation (AF) ablation in the 1990s, the procedure has continuously evolved, with gradual improvements in outcomes and safety. Recent technological advancements include the introduction of contact force catheters and high-resolution electroanatomical mapping systems, while imaging modalities including transesophageal echocardiography and fluoroscopy have become integral parts of AF ablation procedures. Further, intraprocedural intracardiac echocardiography and the integration of cardiac magnetic resonance and computed tomography images with electroanatomical mapping have shown promise to improve procedural outcomes by reducing radiation exposure and procedural times. However, available data on procedural utility and the reduction in AF recurrence rates associated with these modalities are mixed. This review therefore aims to discuss the current common imaging modalities used in AF ablation and their potential impact on outcomes. In particular, imaging is discussed with respect to the important information it offers before, during, and after the procedure. Perspectives on the future of imaging in AF ablation are also shared.
\end{abstract}

KEYWORDS. As low as reasonably achievable (ALARA), computed tomography, electroanatomic mapping, image integration, intracardiac echocardiography, late gadolinium enhancement, magnetic resonance imaging.
ISSN 2156-3977 (print) ISSN 2156-3993 (online) CC BY 4.0 license

(c) 2020 Innovations in Cardiac Rhythm Management

\section{Introduction}

Atrial fibrillation (AF) is the most common form of arrhythmia in the United States and around the world. ${ }^{1}$ Current estimates suggest the number of patients afflicted with AF exceeds 30 million worldwide and three million in the United States. ${ }^{2}$ The economic burden

Dr. Nazarian is a scientific advisor to CardioSolv and Circle Imaging Software and has received research grants from the United States National Institutes of Health, Siemens, ImriCor, and Biosense Webster. The other author reports no conflicts of interest for the published content.

Manuscript received January 30, 2020. Final version accepted August 6, 2020.

Address correspondence to: Saman Nazarian, MD, PhD, Clinical Electrophysiology Section, Cardiovascular Division, Department of Medicine, Perelman School of Medicine at the University of Pennsylvania, 3400 Spruce Street, 9 Founders Pavilion, Philadelphia, PA 19104, USA. Email: saman.nazarian@uphs.upenn.edu. of AF has been clearly established. ${ }^{3}$ The mainstay of AF management includes the prevention of stroke and the introduction of rhythm control strategies involving either antiarrhythmic drugs or catheter ablation. Since the introduction of AF ablation in the 1990s, ${ }^{4}$ the procedure has continuously evolved, with gradual improvements in both outcomes and safety. Recent technological advancements include the introduction of contact force catheters and high-resolution electroanatomical mapping systems, while imaging modalities including transesophageal echocardiography and fluoroscopy have become integral parts of the AF ablation procedure. Similarly, the introduction of intraprocedural intracardiac echocardiography and the integration of cardiac magnetic resonance and computed tomography images with electroanatomical mapping have shown promise in improving procedural outcomes by reducing radiation exposure and procedural times. However, available data on procedural 
utility and the reduction in AF recurrence rates associated with these modalities are mixed. This review aims to discuss the current common imaging modalities used in AF ablation and their potential impact on outcomes. In particular, imaging is discussed with respect to the important information it offers before, during, and after the procedure. Perspectives on the future of imaging in AF ablation are also shared.

AF ablation is effective at improving quality of life and symptoms, particularly in cases where antiarrhythmic drugs have failed. ${ }^{5}$ Techniques have evolved over recent decades with substantial improvements in safety and procedure outcomes. Current guidelines recommend ablation for paroxysmal AF refractory to class I or III antiarrhythmic drugs. ${ }^{5}$ Furthermore, given the improving safety profile and efficacy, there is a class IIa recommendation to conduct ablation prior to the initiation of antiarrhythmic drugs and for persistent AF. Several imaging modalities have been adopted to assist the electrophysiologist with the ablation procedure, each of which assists with different aspects of the procedure, with the overall aim of improving the outcome, safety, or radiation exposure. For instance, transesophageal echocardiography (TEE) is typically employed to rule out left atrial (LA) appendage (LAA) thrombus pre-ablation, intracardiac echocardiography (ICE) enhances the safety of transseptal puncture and catheter tissue contact during ablation, cardiac magnetic resonance imaging (MRI) and computed tomography (CT) help to integrate chamber and pulmonary venous anatomy into the procedural electroanatomic map, and CT imaging alone may be used to rule out atrioesophageal (AE) fistula following ablation if concerning symptoms are noted. Regardless of the imaging modality being used, however, it is important that the electrophysiologist balances the additional information with costs and real value in terms of procedure safety, radiation exposure, and outcomes.

\section{Preprocedural imaging}

One of the most important aspects of AF management is the prevention of LAA thrombus formation by the institution of oral anticoagulation. The incidence of stroke among patients undergoing pulmonary vein (PV) isolation (PVI) has been estimated to be approximately $1.5 \%{ }^{6}$ Data from the surgical literature suggest that $57 \%$ of thrombi in rheumatic AF and 91\% of thrombi in nonrheumatic AF are identifiable in the LAA. ${ }^{7}$ Current guidelines for imaging to rule out LAA thrombus before ablation remain similar to those for electrical cardioversion, with the presence of LAA thrombus being a contraindication to the procedure. The gold-standard modality for the identification of LAA thrombus is TEE. ${ }^{5,8}$ In comparison with TEE, CT imaging showcases excellent negative predictive value but poor specificity and positive predictive value, although the specificity could significantly improve to $99 \%$ with delayed CT. ${ }^{8}$ Cardiac MRI has also been tested for the identification of LAA thrombus in comparison with TEE, with some studies ${ }^{9,10}$ concluding that it, especially when using delayed enhancement sequences, shows comparable performance to that of TEE in identifying LAA thrombi. Advantages of cardiac MRI over CT include a lack of iodinated contrast and radiation exposure. However, CT offers significantly higher spatial resolution, albeit with lower temporal and contrast resolution relative to cardiac MRI. Notably, CT and particularly cardiac MRI are prone to motion artifacts that are often encountered in the cardiac population with arrhythmia and difficulty with breath-holds. In comparison with CT and cardiac MRI, TEE is invasive and associated with an approximately $0.9 \%$ risk of complications such as esophageal perforation, bleeding, sedation-related events, arrhythmia, and even death. ${ }^{10,11}$ For patients unable to undergo TEE due to swallowing difficulties or other gastrointestinal problems, cardiac MRI and CT represent viable options for ruling out LAA thrombus prior to ablation. In contrast, TEE is portable and does not use iodinated or gadolinium contrast agents. However, it is important to note that multiple recent studies have reported that the risk of nephrogenic systemic sclerosis with gadolinium chelates is exceedingly low, especially when new macrocyclic and linear agents are used. ${ }^{12}$ Recently, phased-array ICE has also emerged as a potential adjunct to TEE for LAA thrombus detection. A 2014 study $^{13}$ found that ICE was equally as good as TEE and exhibited potential advantages in identifying LAA thrombus. The best imaging outcome for LAA thrombus with ICE is usually obtained with the ICE catheter located in the pulmonary artery. However, manipulation in the pulmonary artery potentially increases the risk of perforation and requires proficiency with ICE manipulation; thus, more studies are required for further validation of the safety and efficacy of this technique. ${ }^{5}$

Aside from identifying LAA thrombi, preprocedural imaging can provide important anatomic and structural information to assist with the procedure. Reviewing PV anatomy, presence of a patent foramen ovale, anomalous PV drainage or left persistent superior vena cava (SVC), LA size, and LA scar and fibrosis (Figure 1) is important for optimal procedure planning. A lack of detailed understanding of variabilities in PV structure and branching could result in incomplete isolation of all veins or PV stenosis. The most common variations of $\mathrm{PV}$ anatomy described in the literature include left common PV ostium (observed upwards of $83 \%$ of the time), right common PV ostium (seen in up to $40 \%$ of cases), and separate ostium for the right middle vein (affecting up to $27 \%$ of cases).${ }^{14}$ In a study by Toffanin et al. evaluating PV anatomy with TEE and magnetic resonance angiography (MRA), only $42 \%$ of patients showed normal $\mathrm{PV}$ anatomy with two right and left veins. ${ }^{15}$ Measuring the PV diameter size could be essential in procedure planning for cryoablation and is best performed with three-dimensional (3D) imaging modalities such as CT or cardiac MRI. TEE appears to be better able at identifying a patent foramen ovale when compared with CT or cardiac MRI. TEE can also evaluate PV anatomy well, achieving up to $95 \%$ concordance with MRA. ${ }^{15}$ Although TEE tends to underestimate ostial measurements, ${ }^{16}$ image integration with electroanatomic 

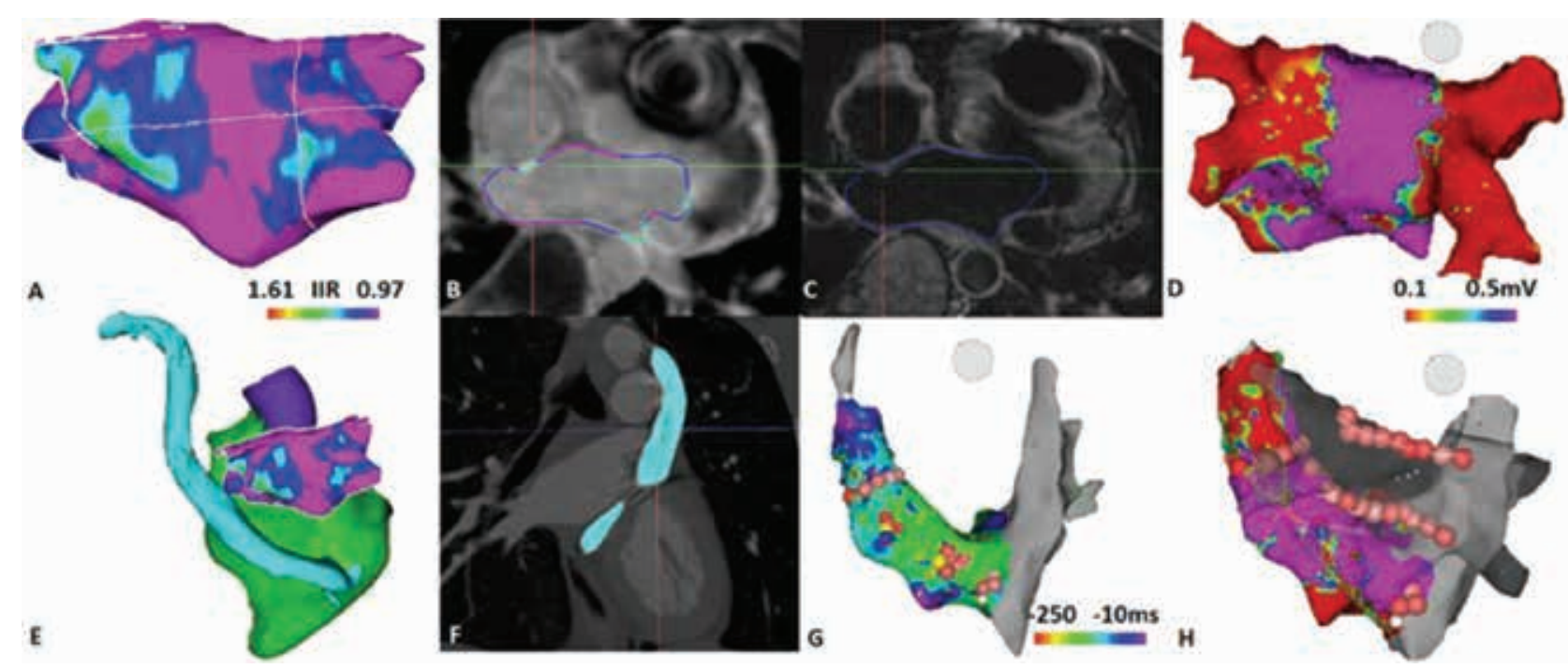

Figure 1: Examples of the utility of image integration for AF ablation. A: CMR-segmented left atrial shell [posteroanterior (PA) view]. The image intensity ratio (IIR) was used to define atrial scar (IIR $>1.61$ correlates with $<0.1 \mathrm{mV}$; IIR $<0.97$ correlates with $>0.5 \mathrm{mV}$ ). The patient underwent prior PVI five months before imaging. B: High-resolution LGE cardiac MRI image (axial view). An atrial scar is noted at the bilateral pulmonary vein antra. C: T2-space cardiac MRI image (axial view). Better identification of the atrial wall of the left atrium is apparent. D: Before ablation, a prior ablation scar at the bilateral PV antra is observable (voltage map, PA view). E: Display of CMR-segmented left atrial scar shell and CT-segmented left-sided SVC. F: Left-sided SVC on CT angiography. G: Non-PV triggers at the ostium of the coronary sinus, proximal coronary sinus, and left-sided SVC (ablation lesions on non-PV triggers, activation map). H: Final ablation lesions on the posterior wall, non-PV triggers, and left-sided SVC isolation (voltage map of left-sided SVC).

mapping requires expertise and can be limited by inaccurate segmentation and image registration. Cardiac MRI is comparable to CT in the evaluation of the $\mathrm{PVs}^{17}$ before AF ablation. Moreover, cardiac MRI remains the gold standard for evaluating fibrosis and scar. Late gadolinium enhancement (LGE) cardiac MRI images of the LA, used to evaluate fibrosis before ablation, have been shown to also predict $\mathrm{AF}$ recurrence following ablation. ${ }^{18,19}$ However, it is important to note that the methods to analyze the extent of LGE vary significantly across centers and result in variable readouts. We have identified and validated two image-intensity standardization methods, image-intensity ratio and z-score which may enhance the homogeneity of data analysis across multiple centers. ${ }^{20,21}$

Other predictors of AF recurrence include LA diameter and volume measurements collected before ablation. These could be obtained readily prior to the ablation procedure with transthoracic echocardiogram (TTE), TEE, CT, or cardiac MRI. Parikh et al. evaluated the performance of LA diameter and volumes using TTE, TEE, and $\mathrm{CT}^{22}$ The study only used diameter measurements from TTE but adopted volume measurements from both TEE and CT. The results confirmed that a larger LA, as measured by either diameter or volume, predicted the recurrence of AF postablation. The use of TEE diameter measurements was more effective than those from TTE and adoption of the TEE volume was superior to the diameter measurement in predicting AF recurrence. Further, however, CT LA volume assessment was superior to TEE volume assessment in predicting AF recurrence. ${ }^{22}$

\section{Intraprocedural imaging}

Image integration. At the time of AF ablation, $\mathrm{CT}$, cardiac MRI, or intraprocedural ICE images can be integrated and registered onto the electroanatomic and/or fast activation map. This practice has been shown to reduce procedural and fluoroscopic times and may improve outcomes. In a study that randomly assigned patients undergoing AF ablation (from 2005-2007) to either image integration with $\mathrm{CT}$ and electroanatomic mapping using the CartoMerge system (Biosense Webster, Diamond Bar, CA, USA) versus the traditional method without integration, ${ }^{23}$ the AF-free survival rate in the integration group was much higher over 14 months \pm 12 months of follow-up ( $88 \%$ versus $69 \%$; $p=0.017)$. A subsequent publication by Bertaglia et al. confirmed the superiority of image integration. ${ }^{24}$ In another registry study using data from patients with paroxysmal AF undergoing ablation in 12 Italian centers, Bertaglia et al. reported improved outcomes with regard to procedure duration and recurrent atrial arrhythmias postablation when image integration with electroanatomic mapping was performed preablation. ${ }^{24}$ Interestingly, fluoroscopic times are generally not lower in such studies; in fact, some studies have reported higher levels of X-ray exposure in the image integration group, ${ }^{23}$ whereas others showed no difference. ${ }^{24}$ It is important to emphasize that these findings have not been consistent. When Caponi et al. examined outcomes postablation in a randomized controlled manner, no difference in disease recurrence or complications was noted when image integration was used. ${ }^{25}$ However, there was a reduction in fluoroscopic duration correlated 
with image integration. The introduction of fast anatomical mapping and multipolar catheters, which occurred after the majority of the above studies were performed, has further led to decreased utilization of image registration during $\mathrm{AF}$ ablation. It is notable, however, that, in the identification of smaller early PV branches, data on proximity to external structures of interest and regions with late enhancement are not provided with fast anatomical mapping. Additionally, the smoothing algorithms of fast anatomic mapping software often create errors at the PV ostia, which have to be subsequently "erased." Without great attention to detail, such errors can direct lesions away from intended ostial targets.

When compared with preprocedural CT or cardiac MRI, intraprocedural ICE offers real-time information such as catheter feedback or the development of effusion prior to clinical manifestations in heart rate and blood pressure and can even identify thrombus formation on catheters during ablation. ${ }^{26,27}$ Furthermore, registration errors from volume shifts are avoided with ICE. ICE has also been shown to improve procedural outcomes and reduce complications in AF ablation. ${ }^{28}$ However, ICE is more likely to miss small proximal branches from PVs, which may be prone to stenosis, as well as other PV anomalies. Thus, we believe a combination of segmented cardiac MRI and live ICE alongside electroanatomic mapping provides the most comprehensive and valuable set of data during $\mathrm{AF}$ ablation.

The effects of radiation exposure in interventional cardiology are well-established. ${ }^{29,30}$ The guiding principle for radiation use in the interventional laboratory, endorsed by major organizations including the Centers for Disease Control and Prevention and the American College of Cardiology, goes by the acronym ALARA ("as low as reasonably achievable"). Further studies are still needed to clearly establish that image integration with preprocedural cardiac MRI reduces the total amount of radiation exposure. Additionally, the use of ICE and electroanatomic mapping can significantly reduce or even eliminate fluoroscopy, as detailed below. It is clear, however, that the total radiation applied to the patient will be increased when preprocedural CT is performed.

Zero-fluoroscopy ablation. Given the mandate of ALARA, the concept of zero fluoroscopy in AF ablation is gaining traction. It is generally accepted that zero radiation is better than any radiation and that no radiation dose is considered safe for the patient, the electrophysiologist, or the cardiac catheterization laboratory staff in general. Orthopedic injuries among interventionalists and electrophysiologists are also well-documented. ${ }^{31}$ Thus, the avoidance of fluoroscopy is appealing provided that the operator is comfortable with adopting alternative guidance methodologies for all procedural components. Electroanatomic mapping and ICE are the main imaging modalities employed for this purpose. The feasibility of zero fluoroscopy was initially shown in 2010. In the study, 20 patients with paroxysmal AF underwent ablation and were followed up with after zero-fluoroscopy AF ablation using the EnSite ${ }^{\mathrm{TM}} \mathrm{Nav}^{\mathrm{TM}}$ mapping system (Abbott Laboratories, Chicago, IL, USA). Transseptal puncture was guided by ICE, while catheter advancement from the lower vessels into the heart was managed by EnSite ${ }^{\mathrm{TM}}$ NavX ${ }^{\mathrm{TM}}$ electroanatomic map guidance. Some patients already had CT imaging segmented and integrated with the EnSite ${ }^{\mathrm{TM}} \mathrm{Nav} \mathrm{X}^{\mathrm{TM}}$ maps, thus improving procedure time. The overall procedure time was longer than that which would be expected for routine AF ablation but, after over six months of follow-up, only $10 \%$ of cases experienced recurrence and all had isolated veins. ${ }^{32}$ Most importantly, there were no complications reported in this first zero-fluoroscopy AF ablation study. Meanwhile, other groups have recently shared their workflow for zero-fluoroscopy AF ablation using the $\mathrm{CARTO}^{\circledR}$ mapping system (Biosense Webster, Diamond Bar, CA, USA) ${ }^{29}$ and image integration with preprocedural $\mathrm{CT}$ or cardiac MRI or intraprocedural 3D ultrasound reconstruction of the LA. Besides the feasibility of zero-fluoroscopy AF ablation procedures, some recent studies have shown that the efficacy, overall procedure duration, and radiofrequency ablation time are not impaired. ${ }^{33}$

\section{Electroanatomic mapping with the use of fast ana- tomic mapping or image integration during atrial fibrillation ablation. The benefits of electroanatomic} mapping during AF ablation with respect to X-ray exposure and procedural time have been established. A prospective randomized trial in 2005 performed by Rotter et al. showed clearly that procedure duration and X-ray exposure were reduced by the technology $y^{34}$ and their findings have been confirmed by subsequent studies. ${ }^{35} \mathrm{An}$ important aspect of most mapping system platforms is the ability to superimpose voltage or activation information upon segmented, registered, and integrated cardiac MRI or CT images, which can enhance procedural guidance. Visualization of abnormal LA bipolar voltage, usually defined as less than $0.5 \mathrm{mV}$, has been associated with extensive LGE on cardiac MRI ${ }^{20}$ and separately with the failure of $\mathrm{AF}$ ablation and increased recurrence rates. ${ }^{36,37}$ Other studies have reported improved outcomes when low-voltage areas, as identified by voltage maps, are targeted for ablation. ${ }^{38,39}$ Large, prospective randomized trials are lacking to confirm improved outcomes in AF ablation based on substrate ablation using voltage mapping with and without the aid of image integration.

\section{Other imaging modalities at the time of atrial fibril-} lation ablation. Image integration with preprocedural CT or cardiac MRI segmentation of the esophagus may be limited in directing ablations away from the esophagus due to small location changes from esophageal peristalsis. ${ }^{40,41}$ Additionally, volume shifts may contribute to errors with electroanatomic map registration. Rotational angiography in the electrophysiology (EP) suite addresses inaccuracies in registration by obtaining $\mathrm{X}$-ray images with the C-arm immediately prior to ablation. These images are 3D-segmented and superimposed either on 
fluoroscopic images or on the electroanatomic map. Some studies have reported reduced radiation doses inherent with rotational angiography in comparison with multislice CT imaging. ${ }^{42}$ However, to circumvent the above problems and to enhance real-time feedback during the procedure while eliminating ionizing radiation, the concept of real-time cardiac MRI for EP procedural guidance was introduced in 2008. ${ }^{43}$ The major advantages of realtime cardiac MRI include providing real-time information on lesion formation and supporting a reduction in fluoroscopy; however, the technology of real-time cardiac MRI in the EP laboratory is still in its infancy. Several centers have reported conducting simple atrial flutter ablations under cardiac MRI guidance ${ }^{44}$ but significant improvements in workflow and noise and image artifact control with suitable electrogram recordings, mapping, and ablation in the strong electromagnetic field associated with this imaging modality have to be resolved before its wide acceptance can proceed.

\section{Postprocedural imaging}

The main purpose of postprocedural imaging in AF is to monitor complications and/or help predict recurrence. The incidence of critical pericardial effusion postablation ranges from $1 \%$ to $1.3 \%{ }^{45}$ In the immediate postprocedural period, ICE could be instrumental in assessing for cardiac perforation and pericardial effusion from ablation. Not so infrequently, patients may be hypotensive immediately after ablation due to sedation changes or as an immediate reaction to protamine for the reversal of anticoagulation. If access is still maintained, ICE can quickly reassure the operator that any finding of hypotension is not due to pericardial effusion. If sheaths have already been removed, then TTE is a viable choice that could rule out effusion with outstanding accuracy. Another possible complication post-AF ablation is phrenic nerve injury, which has an approximate frequency of $1 \%$ with radiofrequency ablation and that of $5 \%$ with cryoablation. In the immediate postoperative period, fluoroscopy with or without a "sniff test" could show elevated hemidiaphragm if concerns about phrenic nerve injury exist.

One of the most dreaded complications of AF ablation is $\mathrm{AE}$ fistula. Although rare, with a reported incidence rate of $0.03 \%$ to $0.08 \%$, it is catastrophic, leading to a greater than $55 \%$ mortality rate in patients who experience the complication. ${ }^{46}$ When AE fistula is not recognized or is addressed only with conservative management, mortality is high. Han et al. showed in a meta-analysis that the median time to presenting with symptoms is approximately 21 days but could range from the day of the procedure to as long as 60 days after the procedure. The symptoms could be variable, with a majority of affected patients presenting with infectious symptoms. Abdominal pain and cardiac symptoms are also common. The imaging modality of choice for evaluating $\mathrm{AE}$ fistula is $\mathrm{CT}$ with contrast. Echocardiography, including TTE and TEE, should be avoided when AE fistula is suspected given the high false-negative rates and clinical deterioration, including strokes, reported when TEE was performed. ${ }^{46,47}$ With prompt identification and surgery, the mortality rate could be improved to approximately $33 \%$, as shown in the largest reported series of cases of AE fistula.

$\mathrm{PV}$ stenosis is another serious complication that can occur after AF ablation. Prior to the advent of wide antral circumferential ablation, PVI consisted mostly of ostial ablation, with a risk of PV stenosis upwards of $42 \%{ }^{48}$ With current technology and wide antral ablation, this risk has been reduced significantly to approximately $0.3 \%$ to $3.4 \%{ }^{48,49}$ If $\mathrm{PV}$ stenosis is suspected after the procedure, cardiac MRI, CT, PV angiography, and TEE are all viable options for diagnosis..$^{50}$

Another potential utility for imaging following AF ablation is to help predict recurrence and the regions of reconnection after PVI. Cardiac MRI studies have shown the regional lack of LGE to predict regions of reconnection during the second procedure after initial cryoablation with reasonable accuracy. ${ }^{51}$ Mishima et al. showed that, when LGE cardiac MRI was performed in patients with recurrent $\mathrm{AF}$ after initial ablation, regions without scar predicted accurately, in $93 \%$ of cases, where reconnections would be noted during EP study at the time of the second procedure. Other research suggests the sensitivity of cardiac MRI seems to be quite limited for gap identification after radiofrequency ablation ${ }^{52}$ and its utility to be minimal following cryoablation. ${ }^{53}$ The extent of LGE on cardiac MRI has been shown to predict ablation success. ${ }^{54}$ McGann et al. performed cardiac MRI before, immediately after, and at three months after AF ablation procedures. Dark, nonenhanced regions noted immediately following the procedure, suggesting no reflow, correlated with locations of scarring three months after surgery and predicted procedural success. Meanwhile, regions of hyperenhancement seen immediately after the procedure represented a continuum of inflammation to necrosis and did not correlate as well with scar at three months of follow-up relative to nonenhanced regions. The nonenhanced regions were shown to demonstrate the no-reflow phenomenon, representing coagulation as well as contraction necrosis.

\section{Summary}

In this review, a variety of image modalities used in AF ablation procedures have been presented. Fluoroscopy, image integration with electroanatomical mapping, and ICE are important adjuncts for AF ablation. Image integration provides the electrophysiologist with detailed anatomic roadmaps with the potential to reduce fluoroscopic exposure and procedural times and possibly improve outcomes. The integration of cardiac MRI, in particular, is advantageous given the lack of radiation exposure or iodinated contrast use in addition to information regarding the presence and amount of fibrosis, which may be crucial for the selection of patients and targets for ablation. With recent studies reporting efficacy and procedure duration outcomes comparable to those of traditional 
methods, zero fluoroscopy will likely gain a significant foothold in the context of AF ablation. The advantages of no fluoroscopy imparted to the patient, staff, and the operator are obvious as no fluoroscopy has been deemed safe and orthopedic injuries to operators in lead aprons are well-documented. Larger randomized controlled trials are needed to definitively answer the question of whether advanced imaging improves procedural success rates in AF. The eagerly anticipated DECAAF II study, which explores whether ablating fibrotic regions improves freedom from $\mathrm{AF}$, is expected to significantly add to our understanding of the importance of advanced imaging in AF ablation.

\section{References}

1. Chugh SS, Havmoeller R, Narayanan K, et al. Worldwide epidemiology of atrial fibrillation: a Global Burden of Disease 2010 Study. Circulation. 2014;129(8):837-847.

2. Colilla S, Crow A, Petkun W, Singer DE, Simon T, Liu X. Estimates of current and future incidence and prevalence of atrial fibrillation in the U.S. adult population. Am J Cardiol. 2013;112(8):1142-1147.

3. Kim MH, Johnston SS, Chu BC, Dalal MR, Schulman KL. Estimation of total incremental health care costs in patients with atrial fibrillation in the United States. Circ Cardiovasc Qual Outcomes. 2011;4(3):313-320.

4. Haissaguerre M, Jais P, Shah DC, et al. Spontaneous initiation of atrial fibrillation by ectopic beats originating in the pulmonary veins. N Engl J Med. 1998;339(10):659-666.

5. Calkins H, Hindricks G, Cappato R, et al. 2017 HRS/EHRA/ ECAS/APHRS/SOLAECE expert consensus statement on catheter and surgical ablation of atrial fibrillation: executive summary. J Arrhythm. 2017;33(5):369-409.

6. Bhargava M, Marrouche NF, Martin DO, et al. Impact of age on the outcome of pulmonary vein isolation for atrial fibrillation using circular mapping technique and cooled-tip ablation catheter. J Cardiovasc Electrophysiol. 2004;15(1):8-13.

7. Blackshear JL, Odell JA. Appendage obliteration to reduce stroke in cardiac surgical patients with atrial fibrillation. Ann Thorac Surg. 1996;61(2):755-759.

8. Lee HG, Shim J, Choi JI, Kim YH, Oh YW, Hwang SH. Use of cardiac computed tomography and magnetic resonance imaging in case management of atrial fibrillation with catheter ablation. Korean J Radiol. 2019;20(5):695-708.

9. Chen J, Zhang H, Zhu D, Wang Y, Byanju S, Liao M. Cardiac MRI for detecting left atrial/left atrial appendage thrombus in patients with atrial fibrillation. Herz. 2018;44(5):390-397.

10. Rathi VK, Reddy ST, Anreddy S, et al. Contrast-enhanced CMR is equally effective as TEE in the evaluation of left atrial appendage thrombus in patients with atrial fibrillation undergoing pulmonary vein isolation procedure. Heart Rhythm. 2013;10(7):1021-1027.

11. Werner N, Zeymer U, Fraiture B, et al. Interventional treatment of paravalvular regurgitation by plug implantation following prosthetic valve replacement: a single-center experience. Clin Res Cardiol. 2018;107(12):1160-1169.

12. Schieda N, Blaichman JI, Costa AF, et al. Gadolinium-based contrast agents in kidney disease: a comprehensive review and clinical practice guideline issued by the Canadian Association of Radiologists. Can J Kidney Health Dis. 2018;5:2054358118778573.

13. Anter E, Silverstein J, Tschabrunn CM, et al. Comparison of intracardiac echocardiography and transesophageal echocardiography for imaging of the right and left atrial appendages. Heart Rhythm. 2014;11(11):1890-1897.

14. Knecht S, Nault I, Wright M, et al. Imaging in catheter ablation for atrial fibrillation: enhancing the clinician's view. Europace. 2008;10(Suppl 3):iii2-iii7.

15. Toffanin G, Scarabeo V, Verlato R, De Conti F, Zampiero AA, Piovesana P. Transoesophageal echocardiographic evaluation of pulmonary vein anatomy in patients undergoing ostial radiofrequency catheter ablation for atrial fibrillation: a comparison with magnetic resonance angiography. $J$ Cardiovasc Med (Hagerstown). 2006;7(10):748-752.

16. Thai WE, Wai B, Truong QA. Preprocedural imaging for patients with atrial fibrillation and heart failure. Curr Cardiol Rep. 2012;14(5):584-592.

17. Hamdan A, Charalampos K, Roettgen R, et al. Magnetic resonance imaging versus computed tomography for characterization of pulmonary vein morphology before radiofrequency catheter ablation of atrial fibrillation. Am J Cardiol. 2009;104(11):1540-1546.

18. Marrouche NF, Wilber D, Hindricks G, et al. Association of atrial tissue fibrosis identified by delayed enhancement MRI and atrial fibrillation catheter ablation: the DECAAF study. $J$ Am Med Assoc. 2014;311(5):498-506.

19. Khurram IM, Habibi M, Gucuk Ipek E, et al. Left atrial LGE and arrhythmia recurrence following pulmonary vein isolation for paroxysmal and persistent AF. JACC Cardiovasc Imaging. 2016;9(2):142-148.

20. Khurram IM, Beinart R, Zipunnikov V, et al. Magnetic resonance image intensity ratio, a normalized measure to enable interpatient comparability of left atrial fibrosis. Heart Rhythm. 2014;11(1):85-92.

21. Kuo L, Zado E, Frankel D, et al. Association of left atrial high-resolution late gadolinium enhancement on cardiac magnetic resonance with electrogram abnormalities beyond voltage in patients with atrial fibrillation. Circ Arrhythm Electrophysiol. 2020;13(2):e007586.

22. Parikh SS, Jons C, McNitt S, Daubert JP, Schwarz KQ, Hall B. Predictive capability of left atrial size measured by CT, TEE, and TTE for recurrence of atrial fibrillation following radiofrequency catheter ablation. Pacing Clin Electrophysiol. 2010;33(5):532-540.

23. Della Bella P, Fassini G, Cireddu M, et al. Image integration-guided catheter ablation of atrial fibrillation: a prospective randomized study. I Cardiovasc Electrophysiol. 2009;20(3):258-265.

24. Bertaglia E, Bella PD, Tondo C, et al. Image integration increases efficacy of paroxysmal atrial fibrillation catheter ablation: results from the CartoMerge Italian Registry. Europace. 2009;11(8):1004-1010.

25. Caponi D, Corleto A, Scaglione M, et al. Ablation of atrial fibrillation: does the addition of three-dimensional magnetic resonance imaging of the left atrium to electroanatomic mapping improve the clinical outcome?: a randomized comparison of Carto-Merge vs. Carto-XP three-dimensional mapping ablation in patients with paroxysmal and persistent atrial fibrillation. Europace. 2010;12(8):1098-1104.

26. Enriquez A, Saenz LC, Rosso R, et al. Use of intracardiac echocardiography in interventional cardiology: working with the anatomy rather than fighting it. Circulation. 2018;137(21):2278-2294.

27. Ren JF, Marchlinski FE, Callans DJ. Left atrial thrombus associated with ablation for atrial fibrillation: identification with intracardiac echocardiography. J Am Coll Cardiol. 2004;43(10):1861-1867.

28. Marrouche NF, Martin DO, Wazni O, et al. Phasedarray intracardiac echocardiography monitoring during 
pulmonary vein isolation in patients with atrial fibrillation: impact on outcome and complications. Circulation. 2003;107(21):2710-2716.

29. Lerman BB, Markowitz SM, Liu CF, Thomas G, Ip JE, Cheung JW. Fluoroless catheter ablation of atrial fibrillation. Heart Rhythm. 2017;14(6):928-934.

30. Limacher MC, Douglas PS, Germano G, et al. ACC expert consensus document. Radiation safety in the practice of cardiology. American College of Cardiology. J Am Coll Cardiol. 1998;31(4):892-913.

31. Goldstein JA, Balter S, Cowley M, Hodgson J, Klein LW, Interventional Committee of the Society of Cardiovascular Interventions. Occupational hazards of interventional cardiologists: prevalence of orthopedic health problems in contemporary practice. Catheter Cardiovasc Interv. 2004;63(4):407-411.

32. Reddy VY, Morales G, Ahmed H, et al. Catheter ablation of atrial fibrillation without the use of fluoroscopy. Heart Rhythm. 2010;7(11):1644-1653.

33. Bulava A, Hanis J, Eisenberger M. Catheter ablation of atrial fibrillation using zero-fluoroscopy technique: a randomized trial. Pacing Clin Electrophysiol. 2015;38(7):797-806.

34. Rotter M, Takahashi Y, Sanders P, et al. Reduction of fluoroscopy exposure and procedure duration during ablation of atrial fibrillation using a novel anatomical navigation system. Eur Heart J. 2005;26(14):1415-1421.

35. Estner HL, Deisenhofer I, Luik A, et al. Electrical isolation of pulmonary veins in patients with atrial fibrillation: reduction of fluoroscopy exposure and procedure duration by the use of a non-fluoroscopic navigation system (NavX). Europace. 2006;8(8):583-587.

36. Verma A, Wazni OM, Marrouche NF, et al. Pre-existent left atrial scarring in patients undergoing pulmonary vein antrum isolation: an independent predictor of procedural failure. J Am Coll Cardiol. 2005;45(2):285-292.

37. Yamaguchi T, Tsuchiya T, Nagamoto $Y$, et al. Long-term results of pulmonary vein antrum isolation in patients with atrial fibrillation: an analysis in regards to substrates and pulmonary vein reconnections. Europace. 2014;16(4):511-520.

38. Yamaguchi T, Tsuchiya T, Nakahara S, et al. Efficacy of left atrial voltage-based catheter ablation of persistent atrial fibrillation. J Cardiovasc Electrophysiol. 2016;27(9):1055-1063.

39. Kottkamp H, Berg J, Bender R, Rieger A, Schreiber D. Box isolation of fibrotic areas (BIFA): a patient-tailored substrate modification approach for ablation of atrial fibrillation. J Cardiovasc Electrophysiol. 2016;27(1):22-30.

40. Njeim M, Desjardins B, Bogun F. Multimodality imaging for guiding EP ablation procedures. JACC Cardiovasc Imaging. 2016;9(7):873-886.

41. Good E, Oral H, Lemola K, et al. Movement of the esophagus during left atrial catheter ablation for atrial fibrillation. J Am Coll Cardiol. 2005;46(11):2107-2110.

42. Li JH, Haim M, Movassaghi B, et al. Segmentation and registration of three-dimensional rotational angiogram on live fluoroscopy to guide atrial fibrillation ablation: a new online imaging tool. Heart Rhythm. 2009;6(2):231-237.

43. Nazarian S, Kolandaivelu A, Zviman MM, et al. Feasibility of real-time magnetic resonance imaging for catheter guidance in electrophysiology studies. Circulation. 2008;118(3):223-229.

44. Mukherjee RK, Chubb H, Roujol S, Razavi R, O'Neill MD. Advances in real-time MRI-guided electrophysiology. Curr Cardiovasc Imaging Rep. 2019;12(2):6.

45. Pedersen MEF, Leo M, Kalla M, et al. Management of tamponade complicating catheter ablation for atrial fibrillation: early removal of pericardial drains is safe and effective and reduces analgesic requirements and hospital stay compared to conventional delayed removal. JACC: Clin Electrophysiol. 2017;3(4):367-373.

46. Han HC, Ha FJ, Sanders P, et al. Atrioesophageal fistula: clinical presentation, procedural characteristics, diagnostic investigations, and treatment outcomes. Circ Arrhythm Electrophysiol. 2017;10(11):e005579.

47. O'Kane D, Pusalkar A, Topping W, Spooner O, Roantree E. An avoidable cause of cardioembolic stroke. Acute Med. 2014;13(3):126-128.

48. Fender EA, Widmer RJ, Hodge DO, et al. Severe pulmonary vein stenosis resulting from ablation for atrial fibrillation: presentation, management, and clinical outcomes. Circulation. 2016;134(23):1812-1821.

49. Dong J, Vasamreddy CR, Jayam V, et al. Incidence and predictors of pulmonary vein stenosis following catheter ablation of atrial fibrillation using the anatomic pulmonary vein ablation approach: results from paired magnetic resonance imaging. J Cardiovasc Electrophysiol. 2005;16(8):845-852.

50. Stavrakis S, Madden GW, Stoner JA, Sivaram CA. Transesophageal echocardiography for the diagnosis of pulmonary vein stenosis after catheter ablation of atrial fibrillation: a systematic review. Echocardiography. 2010;27(9):1141-1146.

51. Mishima T, Miyamoto K, Morita Y, et al. Feasibility of late gadolinium enhancement magnetic resonance imaging to detect ablation lesion gaps in patients undergoing cryoballoon ablation of paroxysmal atrial fibrillation. J Arrhythm. 2019;35(2):190-196.

52. Spragg DD, Khurram I, Zimmerman SL, et al. Initial experience with magnetic resonance imaging of atrial scar and co-registration with electroanatomic voltage mapping during atrial fibrillation: success and limitations. Heart Rhythm. 2012;9(12):2003-2009.

53. Khurram IM, Catanzaro JN, Zimmerman S, et al. MRI evaluation of radiofrequency, cryothermal, and laser left atrial lesion formation in patients with atrial fibrillation. Pacing Clin Electrophysiol. 2015;38(11):1317-1324.

54. McGann C, Kholmovski E, Blauer J, et al. Dark regions of no-reflow on late gadolinium enhancement magnetic resonance imaging result in scar formation after atrial fibrillation ablation. J Am Coll Cardiol. 2011;58:177-185. 